\title{
Satisfação com a imagem corporal e uso de suplementos por frequentadores de academias de ginástica
}

\author{
Satisfaction with body image and use of supplements by gym goers
}

\author{
Lidiane Castro Duarte ${ }^{1}$, Saulo Peters Almas'², Daiane Gonçalves de Oliveira ${ }^{1}$, Sheila Cristina Potente Dutra ${ }^{3}$, \\ Renata Maria Souza Oliveira ${ }^{4}$, Renato Moreira Nunes ${ }^{5}$, Aline Silva de Aguiar Nemer ${ }^{6}$
}

${ }^{1}$ Estudante do Curso de Nutrição do Instituto de Ciências Biológicas da Universidade Federal de Juiz de Fora (UFJF), Juiz de Fora, MG; ${ }^{2}$ Educador Físico graduado pela UFJF, Estudante do Curso de Nutrição do Instituto de Ciências Biológicas da UFJF, Juiz de Fora, MG; ${ }^{3}$ Nutricionista graduada pela Universidade do Estado do Rio de Janeiro (UERJ), Doutora em Fisiopatologia Clínica e Experimental pela UERJ, Professora do Departamento de Nutrição do Instituto de Ciências Biológicas da UFJF, Juiz de Fora, MG; ${ }^{4}$ Nutricionista graduada pela Universidade Federal de Viçosa (UFV), Doutora em Saúde pela UFJF, Professora do Departamento de Nutrição do Instituto de Ciências Biológicas da UFJF, Juiz de Fora, MG; ${ }^{5}$ Nutricionista graduado pela UFV. Doutor em Biologia Celular e Estrutural pela UFV, Professor do Departamento de Nutrição do Instituto de Ciências Biológicas da UFJF, Juiz de Fora, MG; ${ }^{6}$ Nutricionista graduada pela Universidade Federal Fluminense (UFF), Doutora em Patologia pela UFF, Professora do Departamento de Nutrição do Instituto de Ciências Biológicas da UFJF, Juiz de Fora, MG.

Este estudo recebeu auxílio financeiro da Pró-reitoria de Pesquisa da Universidade Federal de Juiz de Fora (PROPESQ/UFJF).

\section{RESUMO}

Objetivos: Verificar o nível de satisfação com a imagem corporal por frequentadores de academias de ginástica e a sua relação com o consumo de suplementos.

Métodos: Realizou-se estudo transversal com frequentadores de academias (praticantes de musculação), com no mínimo seis meses de prática, de ambos os sexos e com idade entre 20 e 40 anos. Os instrumentos utilizados foram: Silhouette Matching Task para a avaliação da percepção da imagem corporal e um questionário semiestruturado para identificar o consumo de suplementos alimentares.

Resultados: Participaram do estudo 45 voluntários, 28 homens e 17 mulheres, com idade média de 27,93 $\pm 6,27$ anos. Trinta e dois participantes (71,1\%) mostravam insatisfação com a imagem corporal, sendo $19(67,9 \%)$ entre os homens e $13(76,5 \%)$ entre as mulheres. Observou-se que as pessoas que consumiam suplementos apresentaram maior satisfação com a imagem corporal do que aquelas que não utilizavam esses produtos. A satisfação com a imagem corporal foi semelhante em pessoas com diferentes tempos de prática de musculação.

Conclusões: Mesmo inseridos em um ambiente fundamentado para a conquista estética e de qualidade de vida, a maioria dos frequentadores de academias encontram-se insatisfeitos com sua imagem corporal. Aqueles que fazem uso de suplementos alimentares possuem menor diferença entre a percepção da imagem atual e a desejada, apresentando maior satisfação com sua imagem corporal.

DESCRITORES: IMAGEM CORPORAL; EXERCÍCIO; SUPLEMENTOS DIETÉTICOS

\section{ABSTRACT}

Aims: To assess the level of satisfaction with body image in gym goers and its relation to the consumption of supplements.

Methods: A cross-sectional study was conducted with gym goers (bodybuilders), with at least six months of practice, of both sexes and aged between 20 and 40 years. The instruments used were: Silhouette Matching Task for the assessment of body image perception and a semistructured questionnaire to identify the consumption of dietary supplements.

Results: The study included 45 volunteers, 28 men and 17 women, with a mean age of $27.93 \pm 6.27$ years. Thirty-two participants (71.1\%) showed dissatisfaction with body image, being 19 (67.9\%) among men and 13 (76.5\%) among women. It was observed that those who consumed supplements had higher satisfaction with their body image than those who did not use these products. Satisfaction with body image was similar in individuals with different times of practicing bodybuilding.

Conclusions: Even inserted in an environment based in aesthetic achievement and quality of life, most gym goers are dissatisfied with their body image. Those who use dietary supplements have less difference between perceived current and desired image, with higher satisfaction with their body image.

KEY WORDS: CORPOREAL IMAGE; EXERCISE; DIETARY SUPPLEMENTS 


\section{INTRODUÇÃO}

A imagem corporal de um indivíduo é definida por aspectos cognitivos, afetivos, sociais/culturais e motores, estando associada com o conceito de si próprio, e influenciada pelas dinâmicas interações entre o ser e o meio em que vive. ${ }^{1} \mathrm{O}$ padrão de corpo magro é o modelo de beleza instituído pela sociedade, sem considerar aspectos relacionados à saúde e constituição física dos indivíduos. ${ }^{2}$ Porém, quando inseridos numa sociedade que estimula este padrão corporal, é provável a procura por alcançar este padrão vigente e considerado "normal" a fim de se enquadrar aos requisitos impostos culturalmente. ${ }^{3}$

Essa procura pela imagem corporal considerada atraente pode levar a transtornos alimentares, como a bulimia e anorexia, os quais podem ter graves consequências, incluindo a morte. ${ }^{4} \mathrm{~A}$ literatura apresenta maior prevalência desses casos em indivíduos que trabalham em profissões em que a estética corporal é considerada um valor agregado, como modelos, bailarinas, atores, atletas e profissionais da área de saúde. ${ }^{5,6}$

Ao enfatizar os frequentadores de academias de ginástica, verifica-se que por ser um local para a prática de exercícios físicos, a academia apresenta-se como um ambiente que favorece a disseminação de padrões estéticos estereotipados, como o corpo com baixa quantidade de gordura e com elevado volume de tônus muscular. ${ }^{7}$ Contudo, o excesso de atividade física pode estar relacionado com riscos à saúde, entre eles os transtornos de imagem corporal. ${ }^{8,9}$

Além disso, a busca por um corpo esteticamente perfeito tem levado a população ao consumo abusivo de substâncias que potencializam em um menor tempo os seus desejos. ${ }^{10}$ Com o mesmo objetivo, os frequentadores de academias têm se submetido ao consumo dessas substâncias, sobretudo de produtos como suplementos alimentares e recursos ergogênicos. ${ }^{11}$ Porém, o excesso de preocupação com a imagem corporal pode acarretar o desenvolvimento da dismorfia muscular, caracterizada como um transtorno da imagem corporal apresentada por atletas e/ou desportistas. ${ }^{7}$

Este trabalho teve como finalidade verificar o nível de satisfação com a própria imagem corporal de frequentadores de academias praticantes de musculação, relacionando-o com o consumo de suplementos alimentares.

\section{MÉTODOS}

Realizou-se um estudo transversal com amostra de conveniência, com indivíduos praticantes de musculação por no mínimo seis meses, com idade entre 20 e 40 anos, de ambos os sexos. O estudo foi realizado em quatro academias de ginástica de Juiz de Fora, estado de Minas Gerais, no período de setembro de 2012 a abril de 2013. O projeto foi aprovado pelo Comitê de Ética da Universidade Federal de Juiz de Fora (Parecer $\left.n^{\circ} 353 / 2011\right)$.

Para avaliação da percepção da imagem corporal utilizou-se a Silhouette Matching Task (SMT), ou Teste de Avaliação da Imagem Corporal, como instrumento de percepção da imagem corporal, ${ }^{12}$ a qual consiste em uma escala com 12 silhuetas numeradas de 1 a 12 , onde a silhueta de número 1 é a mais magra, aumentando consecutivamente a numeração e a forma corporal até a silhueta de número 12. Foi solicitado que o indivíduo assinalasse sua percepção de silhueta atual (SA) e a silhueta que considerava ideal ou desejável (SI ou $\mathrm{SD})$. O nível de satisfação com a imagem corporal foi identificado a partir da diferença entre as silhuetas que os participantes identificaram como atual e desejável (SA - SD) e o percentual de satisfação foi calculado através da fórmula:

$$
\% \text { Satisfação }=\frac{\text { Silhueta atual }- \text { Silhueta desejável }}{12} \times 100
$$

Assim, valores positivos indicaram a vontade de se ter uma silhueta menor, enquanto valores negativos mostraram que a vontade era ter uma silhueta maior que a atual.

Para identificar o consumo de suplementos alimentares e se o participante recebeu orientação para seu uso, foi aplicado um questionário semiestruturado elaborado pela equipe da pesquisa. $\mathrm{O}$ questionário e o Teste de Avaliação da Imagem Corporal foram aplicados com a orientação dos pesquisadores, em sala privada da própria academia.

A normalidade da distribuição da imagem corporal foi realizada através do teste de Shapiro-Wilk. Rejeitando-se a normalidade dos dados, o teste U de Mann-Whitney foi aplicado para a comparação entre aqueles que consumiam ou não suplementos e o teste de Kruskal-Wallis foi utilizado visando à comparação da satisfação com a imagem corporal entre diferentes faixas de tempo de prática de musculação. O critério de significância adotado foi $\mathrm{p}$ menor do que 0,05 . O tamanho de efeito foi calculado através do $r$ de Pearson.

\section{RESULTADOS}

Participaram do estudo 28 homens e 17 mulheres, com idade média igual a $27,93 \pm 6,27$ anos. Trinta e dois 
participantes $(71,1 \%)$ mostravam insatisfação com a imagem corporal, sendo 19 homens $(67,9 \%$ dos 28$)$ e 13 mulheres $(76,5 \%$ das 17$)$. Os praticantes que consumiam suplementos tiveram menor diferença entre a imagem atual e a desejada, não havendo diferenças entre os sexos (Tabela 1).

Quanto às percepções de imagem atual e desejada pelos participantes, verificou-se que 22 participantes do sexo masculino identificaram como silhueta atual as que estavam entre a numeração de 4 a 9 , e como desejável as que estavam entre a numeração de 4 a 8. Entre as mulheres, notou-se uma maior oscilação entre as marcações de silhueta atual e desejável, destacando-se a silhueta 6 como identificação de atual por seis mulheres e desejáveis as que estavam entre a numeração de 1 a 6 por 16 mulheres (Tabela 2).

Tabela 1. Diferença entre a imagem corporal atual e a desejável de acordo com o consumo ou não de suplementos em amostra de 45 frequentadores de academias de ginástica. Juiz de Fora, MG, setembro de 2012 a abril de 2013

\begin{tabular}{|c|c|c|c|c|c|c|c|}
\hline & & & Consome & ementos & & & \\
\hline & & & $=25)$ & & & p* & $\mathbf{r}$ \\
\hline & & Mediana & $\mathrm{IQ}^{* *}$ & Mediana & $\mathrm{IQ}^{* *}$ & & \\
\hline & Homens & 0 & $(-1,5 ; 1,5)$ & -1 & $(-3 ; 0)$ & 0,172 & 0,26 \\
\hline Imagem Corporal & Mulheres & 0 & $(-3,5 ; 0,75)$ & -3 & $(-4,5 ;-2)$ & 0,079 & 0,44 \\
\hline & Total & 0 & $(-2,0 ; 1,0)$ & -2 & $(-3,0 ; 0)$ & 0,015 & 0,36 \\
\hline
\end{tabular}

* Valor p calculado pelo teste de Mann-Whitney.

** IQ: Intervalo interquartil (Quartil 1; Quartil 3).

Tabela 2. Frequência de identificação de silhueta atual e desejável para homens e mulheres. Amostra constituída por 45 frequentadores de academias de ginástica. Juiz de Fora, MG, setembro de 2012 a abril de 2013

\begin{tabular}{|c|c|c|c|c|}
\hline \multirow[b]{2}{*}{$\begin{array}{c}\text { Silhueta apresentada } \\
\text { na escala }\end{array}$} & \multicolumn{2}{|c|}{ Homens $(n=28)$} & \multicolumn{2}{|c|}{ Mulheres $(n=17)$} \\
\hline & $\begin{array}{c}\text { Escolheram como sua } \\
\text { silhueta atual } \\
n(\%)\end{array}$ & $\begin{array}{c}\text { Escolheram como } \\
\text { silhueta desejável } \\
\text { n (\%) }\end{array}$ & $\begin{array}{c}\text { Escolheram como sua } \\
\text { silhueta atual } \\
\text { n (\%) }\end{array}$ & $\begin{array}{c}\text { Escolheram como } \\
\text { silhueta desejável } \\
\text { n (\%) }\end{array}$ \\
\hline 1 & $1(3,57)$ & $2(7,14)$ & $0(0)$ & $2(11,76)$ \\
\hline 2 & $3(10,71)$ & $2(7,14)$ & $2(11,76)$ & $1(5,88)$ \\
\hline 3 & $2(7,14)$ & $2(7,14)$ & $2(11,76)$ & $3(17,65)$ \\
\hline 4 & $6(21,43)$ & $5(17,86)$ & $0(0)$ & $4(23,53)$ \\
\hline 5 & $0(0)$ & $3(10,71)$ & $0(0)$ & $1(5,88)$ \\
\hline 6 & $4(14,29)$ & $6(21,43)$ & $6(35,29)$ & $5(29,41)$ \\
\hline 7 & $3(10,71)$ & $3(10,71)$ & $3(17,65)$ & $1(5,88)$ \\
\hline 8 & $4(14,29)$ & $5(17,86)$ & $0(0)$ & $0(0)$ \\
\hline 9 & $5(17,86)$ & $0(0)$ & $2(11,76)$ & $0(0)$ \\
\hline 10 & $0(0)$ & $0(0)$ & $1(5,88)$ & $0(0)$ \\
\hline 11 & $0(0)$ & $0(0)$ & $1(5,88)$ & $0(0)$ \\
\hline 12 & $0(0)$ & $0(0)$ & $0(0)$ & $0(0)$ \\
\hline
\end{tabular}

Tabela 3. Diferença percentual entre a imagem corporal atual e a desejável de acordo com o tempo de prática de musculação. Amostra constituída por 45 frequentadores de academias de ginástica. Juiz de Fora, MG, setembro de 2012 a abril de 2013

\begin{tabular}{|lccc}
$\begin{array}{l}\text { Tempo de prática } \\
\text { de musculação }\end{array}$ & $\begin{array}{c}\text { Diferença percentual entre a } \\
\text { imagem corporal atual e a desejada }\end{array}$ & \multirow{2}{*}{$\mathbf{p}^{*}$} \\
\cline { 1 - 3 } 6-8 meses & 16,67 & $(-12,5 ; 37,5)$ & 0,097 \\
9-11 meses & 4,17 & $(-12,5 ; 22,92)$ & 0,097 \\
12-35 meses & 25,00 & $(0 ; 37,5)$ & 0,097 \\
Mais de 36 meses & 0 & $(-4,17 ; 0)$ & 0,097 \\
\hline
\end{tabular}

* Valor p calculado pelo teste de Kruskal-Wallis.

** IQ: Intervalo interquartil (Quartil 1 ; Quartil 3).
Observou-se que a satisfação com a imagem corporal não variou entre as diferentes faixas de tempo de prática de musculação (Tabela 3 ).

O consumo de suplementos foi citado por dos 25 $(55,6 \%)$ voluntários. Destes, $14(56,0 \%)$ receberam orientação de algum profissional. $\mathrm{O}$ nutricionista foi o responsável pela orientação em cinco $(35,7 \%)$ casos, enquanto o profissional de educação física orientou oito $(57,1 \%)$ e o médico um $(7,1 \%)$. Entre os $11(44,0 \%)$ que não receberam orientação profissional, nove $(81,8 \%)$ afirmaram consumir suplementos por conta própria e dois $(18,2 \%)$ por orientação de amigos. 


\section{DISCUSSÃO}

O estudo identificou que a maior parte $(71,1 \%)$ dos frequentadores das academias incluídos no estudo estava insatisfeita com sua imagem corporal. Porém os que relataram usar suplementos alimentares apresentaram menor diferença entre a imagem atual e desejável na escala de silhuetas. $\mathrm{O}$ uso de suplementos, utilizados principalmente para proporcionar ganho acelerado de massa muscular ou perda de peso, pode estar relacionado à conquista mais rápida de um “corpo ideal" para esses participantes.

A ingestão de suplementos por praticantes de atividades físicas deve ser constante objeto de estudo, uma vez que o consumo de suplementos nutricionais ou farmacológicos, sem correta prescrição, pode produzir efeitos prejudiciais à saúde, ${ }^{11}$ como sobrecarga do sistema renal e hepático devido à alta carga de nitrogênio, transformação do excesso de proteína em gordura, desidratação em decorrência da produção excessiva de ureia ${ }^{13}$ e contaminação por esteroides sem indicação nos rótulos. ${ }^{14}$ Contudo, fatores como falta de orientação ou orientação prestada por profissionais sem habilitação técnico-profissional, comércio desses produtos na própria academia, falta de legislação rigorosa que não autorize a venda sem receita médica e propaganda da indústria, que lança constantemente no mercado produtos que pressupõem efeitos imediatos e eficazes, ${ }^{10,11}$ contribuem para o crescente número de consumidores desses produtos.

No presente estudo, observou-se consumo de suplementos por mais da metade dos ginastas avaliados, sendo que grande parte das orientações havia sido prestada por profissionais de Educação Física, que mesmo sendo considerados profissionais de saúde pelo Conselho Nacional de Saúde, não são capacitados para receitar medicamentos. ${ }^{15,16}$ Ressalta-se que, quando os atletas restringem sua ingestão energética, usam práticas de perda de peso drásticas, eliminam um ou mais grupos de alimentos, consomem dietas alta em carboidratos com baixa densidade de nutrientes ou com evidências concretas de deficiências nutricionais, aí sim há necessidade de alguma suplementação dietética, que deverá ser feita com a orientação de um nutricionista qualificado.

Os resultados do Teste para Avaliação da Imagem Corporal evidenciam que mesmo quando inseridos em um ambiente fundamentado para a conquista estética e de qualidade de vida, muitos praticantes de atividade física estão insatisfeitos com sua imagem corporal. O mesmo foi identificado em outro estudo realizado com 315 frequentadores de academias, revelando que $48,3 \%$ estavam insatisfeitos com seu corpo. ${ }^{17}$
Verificou-se no presente estudo que para os homens as imagens desejáveis eram na maioria das vezes as silhuetas com numeração acima da marcada como atual, que representam os corpos mais robustos, sendo o mesmo observado em estudo feito com homens da Áustria, França e Estados Unidos, onde a tendência foi a escolha de um corpo mais musculoso como o ideal. ${ }^{18}$ Já para as mulheres, na maioria das vezes o desejo era de um corpo mais magro, sendo normalmente desejável a silhueta com numeração abaixo da identificada como atual, conforme constatado em outros estudos, nos quais as mulheres mostraram preferência pelos corpos mais magros, com menor volume corporal. ${ }^{19,20}$ Esses dados demonstram que homens e mulheres possuem percepções diferentes de seus corpos. O fato de não ter havido diferença entre as imagens corporais dentro de cada sexo pode estar relacionado ao pequeno tamanho amostral, já que o grupo das mulheres, por exemplo, apresentaram diferença maior entre a silhueta ideal e a desejável do que o grupo com todos os participantes.

Algumas limitações foram encontradas no estudo, como a queixa de alguns participantes pela falta de silhuetas que se enquadrassem no desejável para desportistas, como as que exibissem corpos com músculos mais evidentes, revelando a necessidade de adaptação de escalas de avaliação da imagem corporal para o público em questão, visto que praticantes de atividades físicas representam um público vulnerável a transtornos de imagem corporal. ${ }^{7}$ Além disso, foi limitada a participação dos voluntários no estudo o que resultou em um número restrito de amostra, limitando a extrapolação dos resultados.

Apesar das limitações, o estudo mostrou dados preocupantes nessa amostra de desportistas que frequentavam academias por no mínimo seis meses, tempo este suficiente para detectar mudanças corporais com a prática de exercícios. ${ }^{21}$ Além disso, foi possível identificar que não houve diferença estatística significativa conforme o tempo de prática de musculação.

Houve diferença entre os que consumiam ou não suplementos. A menor diferença na percepção de imagem atual e desejável por aqueles que faziam uso de suplementos alerta para uma possível relação entre o uso desses produtos e a conquista acelerada do corpo. A partir dessa constatação, talvez seja possível identificar precocemente transtornos psicológicos e alimentares, como os de comportamento alimentar. Assim, é importante desenvolver estratégias que abordem a temática imagem corporal e uso de suplementos nutricionais. 


\section{AGRADECIMENTOS}

Os autores agradecem à Pró-Reitoria de Pesquisa (PROPESQ) da Universidade Federal de Juiz de Fora pela concessão de bolsas de iniciação científica (BIC-UFJF); a Fabiana Almeida pelo auxílio técnico quanto ao uso dos equipamentos de avaliação nutricional; e a todos os funcionários das academias participantes do estudo.

\section{REFERÊNCIAS}

1. Adami F, Fernandes TC, Frainer DES, Oliveira FR de. Aspectos da construção e desenvolvimento da imagem corporal e implicações na educação física [Internet]. Buenos Aires; 2005; [cited 2013 Jul 10]. Available from: http://www.efdeportes.com/efd83/imagem.htm

2. Oliveira FP, Bosi MLM, Vigário P dos S, Silva VR da. Comportamento alimentar e imagem corporal em atletas. Rev Bras Med Esporte. 2003;9(6):348-56

3. Alves D, Pinto M, Alves S, Mota A, Leirós V. Cultura e imagem corporal. Motri. 2009; 5(1):1-20.

4. Mantovani B, Pinha G, Corrêa B, Bueno L, Freitas M, Damaceno H, Hirai R. Nível de satisfação com a imagem corporal de universitários [Internet]. Buenos Aires 2009; [cited 2013 Jul 10]. Available from: http://www.efdeportes.com/efd132/nivel-de-satisfacao-com-a-imagemcorporal.htm.

5. Salzano FT, Cordás TA. Transtornos alimentares. In: Cordás TA, Salzano FT. Saúde mental da mulher. São Paulo: Atheneu; 2004. p. 201-28.

6. Bosi MLM, Luiz RR, Uchimura KY, Oliveira FP. Comportamento alimentar e imagem corporal entre estudantes de educação física. J Bras Psiquiatr. 2008;57(1):28-33.

7. Lima LD, Moraes CMB, Kirsten VR. Dismorfia muscular e o uso de suplementos ergogênicos em desportistas. Rev Bras Med Esporte. 2010;16(6):427-30.

8. Assunção SSM. Dismorfia muscular. Rev Bras Psiquiatria. 2002;24(3):80-4.

9. Azevedo AMP, Ferreira A de CD, Silva PPC da, Silva EAPC da, Caminha I de O. Dismorfia muscular: características alimentares e da suplementação nutricional. ConScientiae Saúde. 2011;10(1):129-37.

10. Santos MAA, Santos RP. Uso de suplementos alimentares como forma de melhorar a performance nos programas de atividades físicas em academias de ginástica. Rev Paul Educ Fís. 2002;16(2):174-85.

11. Domingues SF, Marins JCB. Utilização de recursos ergogênicos e suplementos alimentares por praticantes de musculação em Belo Horizonte, MG. Fit Perf J. 2007;6(4):219-26.

12. Marsh HW, Roche LA. Predicting self-esteem from perceptions of actual and ideal ratings of body fatness: is there only one ideal “supermodel". Res Q Exerc Sport. 1996;67(1):13-23.

13. Larosa G. Dieta hiperproteica. Fit Perf J. 2006;5(3):189-90.

14. Terada LC, Godoi MR de, Silva TCV, Monteiro TL. Efeitos metabólicos da suplementação do whey protein em praticantes de exercícios com pesos. Rev Bras Nut Esp. 2009; 3(16):295-304

15. Lei Federal $n^{\circ} 9.696$, de $1^{\circ}$ de setembro de 1998, artigo III.

16. Conselho Nacional de Saúde. Resolução Federal no 218 de 6 de março de 1997.

17. Tessmer CS, Silva MC, Pinho MN, Gazalle FK, Fassa AG. Insatisfação corporal em frequentadores de academia. Rev Bras Ci e Mov. 2006;14(1):7-12

18. Pope HG Jr, Gruber AJ, Mangweth B, Bureau B, deCol C, Jouvent R, Hudson JI. Body image perception among men in three countries. Am J Psychiatry. 2000;157(8):1297-301.

19. Cachelin FM, Rebeck RM, Chung GH, Pelayo E. Does ethnicity influence body-size preference? A comparison of body image and body size. Obes Res. 2002;10(3):158-66.

20. Damasceno VO, Perrout LJR, Vianna JM, Vianna VRA, Novaes JS. Tipo físico ideal e satisfação com a imagem corporal de praticantes de caminhada. Rev Bras Med Esporte. 2005;11(3):181-6.

21. Annesi JJ. Relations of changes in self-regulatory efficacy and physical self-concept with improvements in body satisfaction in obese women initiating exercise with cognitive-behavioral support. Body Image. 2010;7(4):356-59. 